\title{
Intervenciones basadas en la evidencia para mejorar los cuidados paliativos del dolor, la disnea y la depresión en el final de la vida
}

Evidence based interventions to improve the palliative care of pain, dyspnea and depression at the end of life

Qaseem A.Ann Intern Med.2008:148:141-146.

Las recomendaciones que siguen son para ser implementadas durante la atención de pacientes con enfermedades serias al final de la vida. Todas son fuertes y, excepto la quinta recomendación, cuentan con un respaldo provisto por evidencia de moderada calidad.

1) Evaluar regularmente sobre la presencia de dolor, disnea $y$ depresión (recomendación fuerte con respaldo provisto por evidencia de moderada calidad).

2) Usar terapias de efectividad probada para el manejo del dolor. En pacientes con cáncer incluir anti inflamatorios no esteroides (AINE) opioides y bifosfonatos (recomendación fuerte con respaldo provisto por evidencia de moderada calidad).

3) Usar terapias de efectividad probada para el tratamiento de la disnea como los opioides en pacientes con disnea refractaria a otros tratamientos y la oxígeno-terapia para el alivio de corto plazo de la hipoxemia (recomendación fuerte con respaldo provisto por evidencia de moderada calidad).

4) Usar terapias de efectividad probada para el tratamiento de la depresión. Para los pacientes con cáncer pueden conside- rarse los antidepresivos tricíclicos, los inhibidores selectivos de la recaptación de serotonina y las intervenciones psicosociales (recomendación fuerte con respaldo provisto por evidencia de moderada calidad).

5) Asegurarse de ir anticipándose en el planeamiento de los cuidados, lo que incluye interrogar al paciente sobre las decisiones que él desee que se tomen en momentos avanzados de su enfermedad y/o durante el final de su vida (recomendación fuerte con respaldo provisto por evidencia de baja calidad).

Resumido de Qaseem A y col. Evidence-Based Interventions to Improve the Palliative Care of Pain, Dyspnea, and Depression at the End of Life: A Clinical Practice Guideline from the American College of Physicians. Ann Intern Med. 2008; 148:141-146. Disponible en URL: http://www.annals.org/cgi/reprint/148/2/141.pdf

Palabras claves: guía de práctica clínica, cuidados paliativos. Keywords: clinical guideline, palliative care. Fuentes de financiamiento: American College of Physicians.
Comentario

Los cuidados paliativos oncológicos, no oncológicos y del fin de la vida suponen una amplia gama de contenidos personalizados para cada enfermo y su familia; requiriendo cada caso una detallada evaluación previa a la implementación de las diferentes intervenciones, y reconociéndose la dificultad para reunir evidencia ${ }^{1}$ avalada por estudios bien diseñados y controlados. Por otro lado, no existe evidencia que respalde cuando es "el momento" óptimo para ofrecer a un paciente y a su familia los servicios de un equipo de cuidados paliativos ${ }^{2}$, por lo que se recomienda ofrecerlos sobre la base los síntomas y las preferencias. La experiencia indica que aunque cada situación requiere una evaluación individualizada, existen problemáticas compartidas por estos pacientes y familias que suele ser necesario abordar y que pueden resumirse en: dolor y otros síntomas (disnea, constipación, anorexia, etc.), el bienestar psicológico(niveles de afrontamiento, adaptación a la enfermedad), coordinación y planificación anticipada de los cuidados y estrés en cuidadores.

Respecto de su eficacia para el tratamiento del dolor un metanálisis ${ }^{3}$ que había incluido 43 artículos reportó que la intervención de un equipo de cuidados paliativos se asocia a un mejor control del dolor con un tamaño del efecto de 0,13 (IC95\% 0,11 a 0,63) desvíos estándar en los puntajes de dolor. En cuanto a la disnea, una revisión sistemática que había incluido 13 estudios ${ }^{4}$, demostró la eficacia de la morfina para el alivio de la disnea en pacientes con enfermedad pulmonar avanzada con una diferencia media estandarizada de $-0,3$ (IC95\% -0,50 a $-0,13)$.

Por lo tanto, se mantiene la vigencia de los opioides ${ }^{5}$ los adyuvantes para el alivio del dolor oncológico; de los antidepresivos en pacientes con una sobrevida estimada mayor de seis semanas y el abordaje inter y multidisciplinario longitudinal y continuo. Este tipo de estrategia aporta una diversidad de intervenciones difíciles de medir objetivamente (tiempo que deben ser empleadas, efectividad, eficacia, etc.) tanto para los familiares como para el equipo de salud. Vale aclarar que existe insuficiente evidencia para respaldar el uso de medicinas complementarias para el alivio del dolor oncológico y de la disnea.

\section{Conclusiones de los comentadores}

Continúan recomendados los opioides y los adyuvantes para el control de dolor oncológico y de la disnea; la oxigenoterapia y la rehabilitación respiratoria para los pacientes EPOC; el tratamiento con antidepresivos y psicoterapia en individuos con depresión; y mantener el abordaje multidisciplinario, la continuidad de los cuidados y la planificación anticipada, para todos los pacientes y familias.

Gloria Vega [ Medica de Familia Unidad de Cuidados Paliativos del Hospital Enrique Tornú, Buenos Aires, Argentina ]

Sergio Terrasa [ Senvicio de Medicina Familiar y Comunitaria del Hospital Italiano de Buenos Aires ]

Vega G y Terrasa S. Existe evidencia para respaldar intervenciones paliativas y cuidados durante el fin de la vida. Intervenciones basadas en la evidencia para mejorar los cuidados paliativos del dolor, la disnea y la depresión en el final de la vida. Evid. Act. Pract. Ambul. 12(2). 51. Abr-Jun. 2009 Comentado de: Lorenz K y col. Evidence for improving Palliative Care at the End of life: a Sistematic Review. Ann Intern Med. 2008; 148:147159.

\section{Referencia}

1. Rodríguez L. Dificultades para la generación de evidencia sobre la efectividad de los cuidados paliativos. Evid. actual. práct. ambul; 11(6):167, Nov-Dic 2008. Comentado de: Zimmermann C y col. Effectiveness of Specialized Palliative Care: A Systematic Review. JAMA. 2008; 299(14):1698-1709. Disponible en URL: http://www.foroaps.org/files/dific\%20para\%20la\%20generac.pdf

2. Qaseem A y col. Evidence-Based Interventions to Improve the Palliative Care of Pain, Dyspnea, and Depression at the End of Life: A Clinical Practice Guideline from the 2. Qaseem A y col. Evidence-Based Interventions to Improve the Pallatican College of Physicians. Ann Intern Med. 2008; 148:141-146.

American College of Physicians. Ann Intern Med. 2008; 148:141-146. the National Assembly of Wales. London: Department of Palliative Care and Policy, Velindre NHS Trust, and Department of General Practice, University of Wales College of Medicine; 2000.

4. Jennings A y col. A systematic review of the use of opioids in the management of dyspnoea.Thorax.2002;57:939-44. 5. Adyuvant analgesics in pain management. Oxford Texbook of Palliative Medicine. 2007; 354-365. 